\title{
Bioanalysis
}

\section{Extending flow cytometry sample stability by freezing lysed whole blood for clinical monitoring of Treg}

\author{
Jing Yu*, ${ }^{*}$, Susmita Jasti**, ${ }^{\text {, Deanna Mason }}{ }^{1}$ \& Amanda Hays ${ }^{1}$ (iD \\ ${ }^{1}$ PRA Health Sciences, 10836 Strang Line Road, Lenexa, KS 66215, USA \\ *Author for correspondence: Tel.: +1 913345 5719; yujing@prahs.com \\ **Author for correspondence: Tel.: +1 913345 5752; jastisusmita@prahs.com
}

\begin{abstract}
Aim: A major challenge for flow cytometry assays supporting clinical trials is postcollection sample stability. Here we present an approach that could mitigate the stability issue while preserving sample integrity and cellular markers, especially when enumerating rare populations such as Tregs. Materials \& methods: Stability was evaluated using whole blood stored at room temperature and lysed whole blood stored at $-80^{\circ} \mathrm{C}$. Results: Freezing of lysed whole blood preserved sample integrity and prolonged sample stability for Treg percentage, absolute cell count and median fluorescent intensity values to 11 versus 3 days at room temperature storage. Conclusion: Frozen storage of lysed whole blood can extend sample stability, improve data quality and facilitate sample batch processing during clinical study sample analysis.
\end{abstract}

First draft submitted: 1 April 2020; Accepted for publication: 20 May 2020; Published online: 3 June 2020

Keywords: flow cytometry • FOXP3 • lysed frozen whole blood • Treg • sample stability

Tregs are a distinct subset of $\mathrm{CD}^{+} \mathrm{T}$ cells that play a crucial role in immune tolerance and are essential for the maintenance of immune homeostasis [1]. Tregs not only suppress conventional $\mathrm{T}$ cells but also other cell types such as B cells, natural killer cells, dendritic cells and monocytes via direct and indirect mechanisms [2]. These underlying processes by which Tregs maintain tolerance can be appreciated from multiple studies pertaining to defective functions of Tregs [3]. These studies have clearly depicted their role in impaired immune responses and autoimmunity, thus, highlighting Treg's therapeutic potential. Mostly, clinical trials relevant to Tregs are based on either adoptive transfer of expanded Tregs [4,5] or treatment regimens that indirectly affect the levels of Tregs in patient cohorts such as low dose IL-2 or IL-2 conjugate treatment [6,7]. The success of the latter method may be in part dependent on assays in which the Treg's population is monitored from whole blood using flow cytometry.

Flow cytometry is a versatile tool that is being profoundly used to support drug development programs. However, the major challenge that the biopharma and clinical research organizations face when developing assays to support clinical trials is postsample collection whole blood stability [8]. Therefore during method development, it becomes imperative to establish sample stability that is in turn dependent on factors such as the specimen type, assay design, the choice of anticoagulant and sample storage conditions [9]. Furthermore, the sample logistics are very complicated as there is a need to consider the time taken to ship the samples from the clinical investigation sample collection sites to the testing facility and the operational requirement to batch process samples. Although several whole blood stabilization products (e.g., Cyto-Chex BCT and Transfix) extend sample stability, these may not be suitable for all leukocyte populations possibly due to their ability to denature certain epitopes $[8,9]$, leading to suboptimal detection of certain cellular marker expression. In addition, conflicting results have been documented where these products have failed to provide stability for delayed analysis $[10,11]$.

In this article, we present a method that we routinely perform in our laboratory that mitigates the above mentioned issues; especially when studying rare populations such as Tregs. Depending on Treg biomarker stability, whole blood samples can be lysed upon collection at the clinical site and shipped frozen to the testing laboratory for batch analysis, or shipped at ambient temperature to the testing laboratory for lyse freeze and batch analysis. The first option has 
been applied successfully to support a previous Treg clinical trial in our laboratory. The latter option is described in this manuscript.

\section{Materials \& methods}

Peripheral blood samples from healthy donors $(\mathrm{n}=3)$ were collected according to ethical guidelines. Informed consent was obtained from each volunteer. Sodium heparin collection tubes (BD, NJ, USA) were used in all experiments unless indicated otherwise. To evaluate stability of lysed whole blood, $200 \mu \mathrm{l}$ of samples were lysed with $2 \mathrm{ml}$ of $1 \mathrm{X}$ BD FACS lysing solution (BD) at room temperature (RT) for $15 \mathrm{~min}$. Lysed samples were immediately flash frozen and then stored at $-80^{\circ} \mathrm{C}$ for predefined stability time periods. The lysed frozen samples were removed from $-80^{\circ} \mathrm{C}$ storage and thawed at $37^{\circ} \mathrm{C}$. Immediately after thawing, samples were centrifuged and washed using Stain Buffer (FBS; BD PharMingen, CA, USA). Samples were then treated with Human TruStain FCX Fc receptor blocking solution (BioLegend) followed by staining with fluorochrome-conjugated antibodies against surface markers CD3 (PerCP-Cy5.5), CD4 (BV510), CD25 (BB515), CD127 (BV421) and CD45RO (PE-Cy7) (BD). Samples were fixed and permeabilized using Human FoxP3 Buffer set (BD) according to manufacturer's recommendation. After permeabilization, samples were washed and stained using fluorochromeconjugated antibodies against intracellular markers FOXP3 (AF647) and Helios (PE; BD). Finally samples were washed and resuspended in Stain Buffer prior to acquisition. Baseline and whole blood samples stored at RT were processed in the same manner except not subjected to flash freeze and thawing. Stability assessments were carried out at baseline (day 0 ) and after 1, 2 and 3 days of storage (whole blood at RT and lysed frozen whole blood at $-80^{\circ} \mathrm{C}$ ). Extended stabilities of lysed frozen whole blood were evaluated after 7 and 11 days of storage at $-80^{\circ} \mathrm{C}$.

Samples were acquired on BD FACSCanto II (BD) instrument. BD Cytometer Setup and Tracking beads (BD) and SPHERO Ultra Rainbow Calibration Particle Kit (Spherotech, IL, USA) were used for flow cytometer daily calibration and instrument standardization. Compensation setup was performed on each day of analysis using UltraComp eBeads compensation beads (Thermo Fisher Scientific, MA USA). Spectral overlap values were calculated using FACSDiva software (BD). Flow cytometry data were analyzed using FCS Express IVD software (De Novo Software, CA USA). Absolute count for lymphocytes was determined using Sysmex XS-1000i hematology analyzer (Sysmex, IL, USA) prior to whole blood lysis. Absolute counts (cells per $\mu$ l) for Tregs and subset populations were calculated based on lymphocyte absolute count and relative percentage to lymphocytes. GraphPad Prism 8.2.0 was used to plot graphs. Percent change from baseline was calculated as [(Stability time point - Baseline) / Baseline]*100.

This research was conducted according to the principles of the Declaration of Helsinki.

\section{Results \& discussion}

Optimized panel design supports sodium heparin as the preferred matrix

Tregs are a rare cell population that accounts for $5-10 \%$ of all circulating $\mathrm{CD} 44^{+} \mathrm{T}$ cells [2]. Due to their low occurrence in peripheral blood, it is crucial to design a panel consisting of a set of markers with appropriate fluorochrome assignments that not only identifies Treg populations but also delivers optimal discrimination between subset populations. Tregs are commonly identified as $\mathrm{CD} 3^{+} \mathrm{CD} 4^{+} \mathrm{CD} 25^{\mathrm{pos} / \mathrm{high}} \mathrm{CD} 127^{\text {neg } / \text { low }}$ cells, and can be further characterized through expression of FOXP3, Helios, Ki67, PD-1, CTLA-4, ICOS, CD45RO, CD45RA and other functional markers [12-16]. The marker selection in the current panel was based on client's clinical study needs together with literature evidence such as importance of FOXP3 expression for Treg lineage maintenance [1]; clinical applications of $\mathrm{CD} 4{ }^{+} \mathrm{CD} 25^{\text {pos }} \mathrm{CD} 127^{\text {low }}$ Tregs in regulatory T-cell therapy [5,17]; expression of CD45RO on memory T cells, Tregs and activated Tregs [4,16,17]; and association of Helios with FOXP3 to maintain suppressive function [18]. Fluorochrome assignment to individual markers was based on gating strategy, specific cytometer configuration, fluorochrome property, resolution impact matrix and antigen expression pattern. The final panel design and gating strategy are shown in Figure 1A. Following exclusion of nonlymphocytes and doublets, cells are gated as $\mathrm{CD} 3^{+} \mathrm{CD} 4^{+} \mathrm{T}$ cells, total Tregs $\left(\mathrm{CD} 3^{+} \mathrm{CD} 4^{+} \mathrm{CD} 25^{\text {pos } / \text { hi }} \mathrm{CD} 127^{\text {neg/low }}\right.$, from here on referred as Tregs), FOXP3 ${ }^{+}$Tregs $\left(\mathrm{CD}^{+} \mathrm{CD} 4^{+} \mathrm{CD} 25^{\text {pos } / \mathrm{hi}} \mathrm{CD} 127^{\text {neg }} /\right.$ low $F O X P 3^{\text {pos }} / \mathrm{hi}$; excluding FOXP3 $\left.{ }^{\mathrm{dim}}\right)$ and Helios $^{+} \mathrm{FOXP}^{+}$Tregs $\left(\mathrm{CD} 3^{+} \mathrm{CD}^{+} \mathrm{CD} 25^{\mathrm{pos} / \mathrm{hi}} \mathrm{CD} 127^{\text {neg } / \text { low }} \mathrm{FOXP} 3^{\text {pos } / \text { hi }}\right.$ Helios +$)$. CD $45 \mathrm{RO}$ expression was then evaluated in each of the above four cell populations. The plot for CD45RO expression in FOXP3 ${ }^{+}$Tregs is shown in Figure 1 and corresponding data are included in subsequent Figure 3. Plots and data for CD45RO expression in the other three cell populations are not shown in this manuscript. 

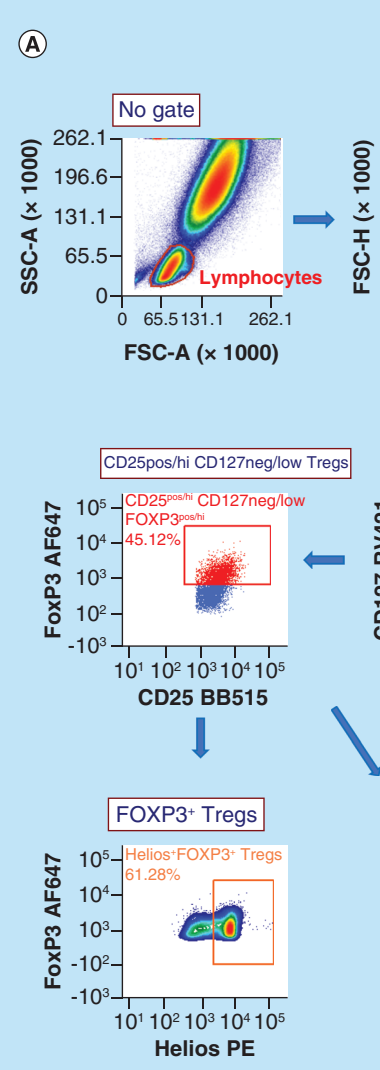
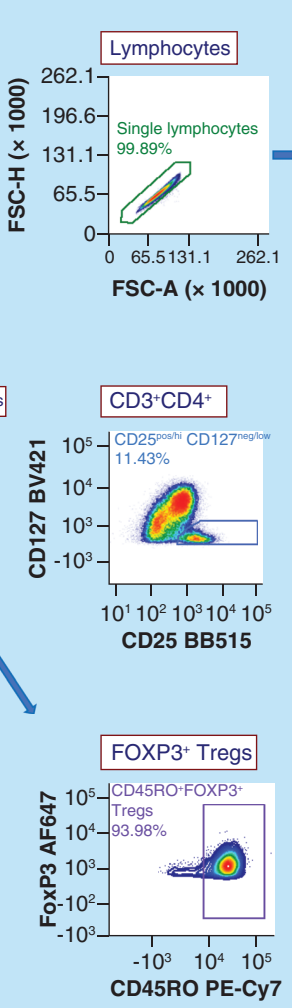
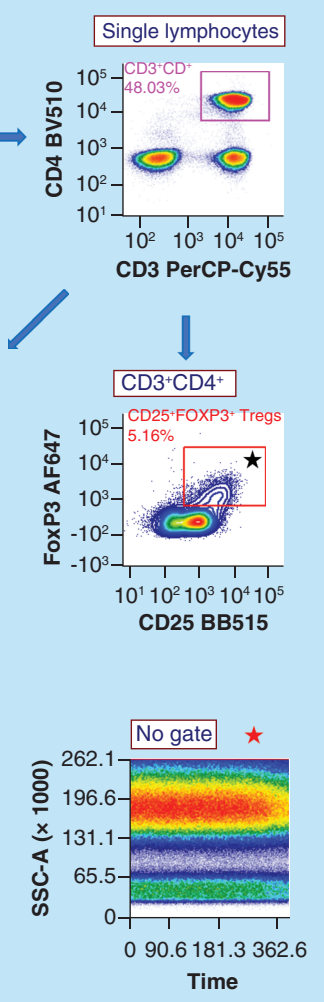

(B)
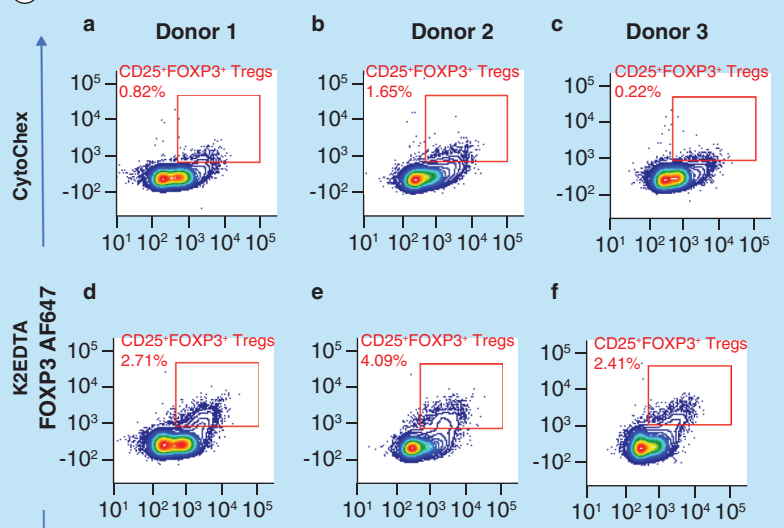

f
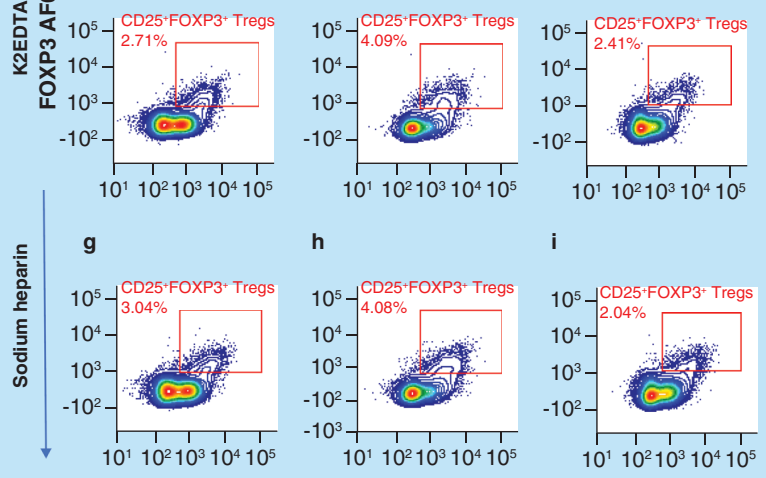

h

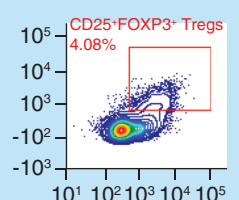

i

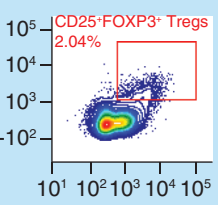

CD25 BB515

Figure 1. Key method development parameters for the Treg flow assay: panel design, gating strategy and matrix selection. (A) Panel design and gating strategy for the Treg assay. Populations mentioned on top of each plot indicate the parent population. Arrows denote gating hierarchy. The gate placement for $\mathrm{CD} 25^{\mathrm{pos} / \mathrm{hi}} \mathrm{CD} 127^{\text {neg/low }} \mathrm{FOXP3} 3^{\mathrm{pos} / \mathrm{hi}}$ population is based on the $\mathrm{CD} 25^{+} \mathrm{FOXP3^{+ }}$ Tregs gate (excluding FOXP3 ${ }^{\mathrm{dim}}$ ) indicated by the black star. The red star denotes a time plot used only to monitor the flow of events. (B) Comparison of FOXP3 ${ }^{+}$Treg staining from whole blood samples collected in Cyto-Chex (a-c), K2EDTA (d-f) and sodium heparin $(\mathbf{g}-\mathbf{i}), \mathrm{n}=3$.

Since the choice of anticoagulant influences sample stability [8], we evaluated Cyto-Chex, EDTA, and sodium heparin as candidate sample collection matrices to support optimal assay performance and sample stability. Traditionally, Cyto-Chex blood collection tubes have been successfully used in flow cytometry to extend sample stability, especially for delayed immunophenotyping [19]. However, as shown in Figure 1B a-c, Cyto-Chex fixation of whole blood was associated with drastically low percentages of the Treg population together with significantly decreased signal intensity of FOXP3. The staining pattern was consistent in all three donors. On the contrary, both EDTA and sodium heparin matrices gave similar results for $\mathrm{FOXP}^{+}$Treg populations in all three donors (Figure 1B $\mathrm{d}-\mathrm{i})$. To further decipher the appropriate choice of anticoagulant, we performed a preliminary stability comparison $(0-48 \mathrm{~h})$ between samples collected in sodium heparin and EDTA blood collection tubes. We observed that RT stored sodium heparin whole blood provided better stability than EDTA whole blood stored at either $4^{\circ} \mathrm{C}$ or RT (data not shown). Consequently, sodium heparin whole blood was confirmed to be the 'choice of matrix' that was investigated further in stability experiments.

\section{Lysed frozen whole blood preserves light scatter properties of leukocytes}

Sample stability evaluation was performed as described in the Materials \& methods section. Data analysis included both visual inspection as well as statistical evaluation of changes of stored samples from the baseline. Figure 2 shows the comparison of the representative scatter plots between the baseline sample and samples that were either stored at RT or lysed and stored frozen at $-80^{\circ} \mathrm{C}$. When compared with baseline samples (Figure 2A), the RT stored samples showed degradation of the granulocyte population over time from day 1 to 3 (Figure 2B-D). These results were not unexpected as light scatter properties of granulocytes may be impacted due to increasing degranulation during storage at RT [9]. As evident from Figure 2B-D, the degraded granulocytes are in juxtaposition with the 


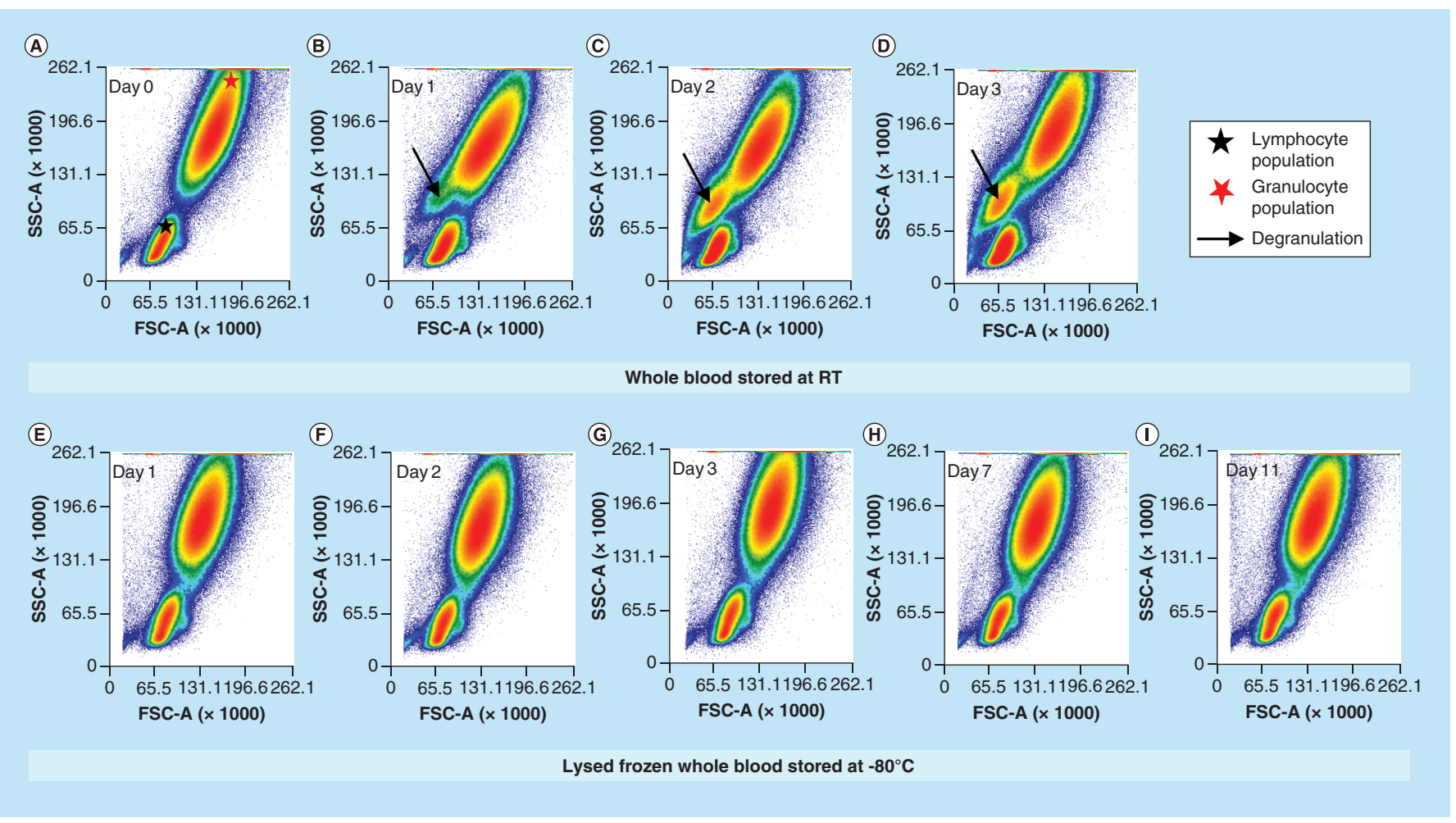

Figure 2. Comparison of scatter plot profiles to determine sample integrity. Forward scatter and side scatter profiles were evaluated for whole blood samples stored at RT from day 1 to 3 (B-D) and lysed frozen whole blood samples from day 1 to 11 (E-I). Day 0 samples served as baseline (A). $n=3$, scatter plots from one representative donor are shown.

RT: Room temperature.

lymphocytes, and this may further raise the possibilities of cross contamination of the latter population. The extent of contamination may or may not be evident just based on the visual inspection of the scatter plots as there may be a risk of nonspecific binding of these cells to the populations down in hierarchy. Therefore, even though the lymphocyte population appeared to be less impacted, we decided the benefits to explore an alternative storage method to improve sample integrity and biomarker data quality. As shown in Figure 2E-G, the lysed frozen whole blood displayed strikingly similar scatter profiles to the baseline sample. Furthermore, the light scatter properties were comparable to baseline at extended time points (Figure $2 \mathrm{H}-\mathrm{I}$ ). These scatter profiles from day 1 to 11 illustrated a nice demarcation between the granulocytes and lymphocyte populations, thus, resulting in the ease of gating. Collectively, these data suggested that red blood cell lysis and frozen storage of lysed whole blood samples prior to staining help to preserve the scatter profiles of leukocyte populations as compared with samples stored at RT.

Lysed frozen whole blood demonstrates extended stability in cell percentages, median fluorescent intensity values \& absolute counts of Tregs \& subsets

In order to compare the stability of whole blood at RT and lysed frozen whole blood at $-80^{\circ} \mathrm{C}$, cell percentages, median fluorescent intensity (MFI) values and absolute cell counts of Tregs and subsets were analyzed and changes from baseline were calculated. Figure 3A-D illustrates cell percentage change from baseline for CD25 pos/hi $C D 127^{\text {neg/low }}$ Tregs (Tregs $\%$ ), FOXP3 ${ }^{+}$Tregs $\%$, Helios ${ }^{+} \mathrm{FOXP}^{+}{ }^{+}$Tregs $\%$ and $\mathrm{CD} 45 \mathrm{RO}^{+} \mathrm{FOXP}^{+}{ }^{+}$Tregs $\%$, respectively, at different postcollection time points for whole blood samples stored at RT. As evident from Figure 3A, Tregs appeared to be stable initially but later showed a continuous gradual decline in cell percentages. The pattern was consistent in all three donors with two of the donors displaying $\geq 20 \%$ change from baseline by day 3 . FOXP $3^{+}$Tregs $\%$ was more stable with change from baseline within $20 \%$ up to day 3 . Helios ${ }^{+} \mathrm{FOXP} 3^{+}$Tregs $\%$ showed a variable trend at day 3 among different donors. $\mathrm{CD} 45 \mathrm{RO}^{+} \mathrm{FOXP} 3^{+}$Tregs $\%$ also showed an apparent slow decrease in stability over time with one of the three donors displaying near $20 \%$ change from baseline by day 3 . Conversely, better stability 


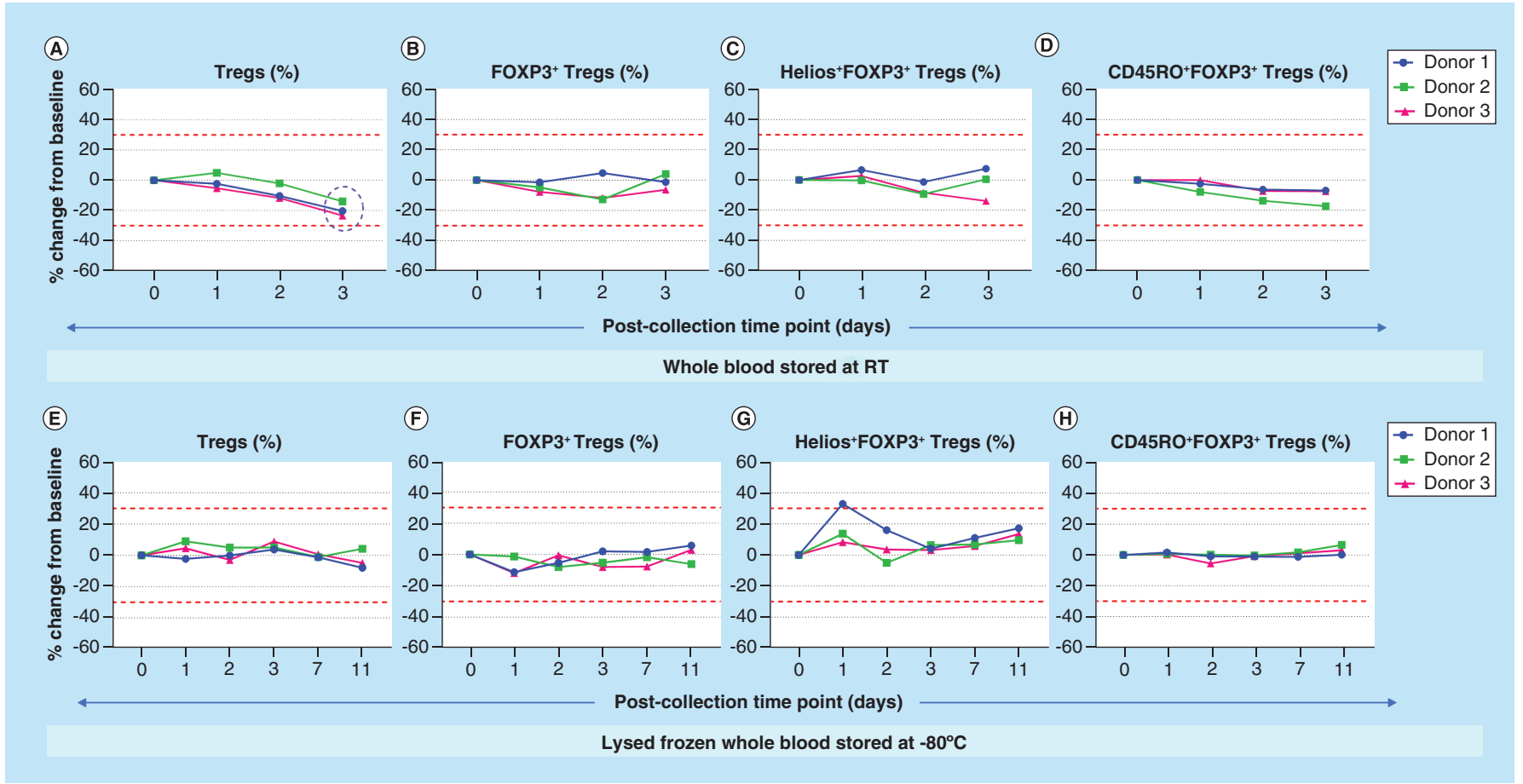

Figure 3. Comparison of stability profiles of percentages of Tregs and subsets. Stability was evaluated for whole blood samples at RT and lysed whole blood at $-80^{\circ} \mathrm{C}, \mathrm{n}=3$. Graphs show percent change from baseline for Tregs and subsets at RT (A-D) and at $-80^{\circ} \mathrm{C}(\mathrm{E}-\mathrm{H})$. The $\mathrm{X}$-axis represents postcollection time points. Top and bottom red lines on each graph indicate $+30 \%$ and $-30 \%$ change from baseline, respectively. Purple circles indicate change from baseline $\geq 20 \%$.

RT: Room temperature.

profiles were observed for Tregs $\%$, FOXP3 ${ }^{+}$Tregs $\%$, Helios ${ }^{+} \mathrm{FOXP}^{+}{ }^{+}$Tregs $\%$ and $\mathrm{CD} 45 \mathrm{RO}^{+} \mathrm{FOXP}^{+}{ }^{+}$Tregs $\%$ in lysed frozen whole blood samples as shown in Figure 3E-H. On day 3 of frozen storage, Tregs and all three subsets of cell percentages showed less than $10 \%$ change from baseline in all three donors. As we further evaluated the frozen stability beyond day 3 , Tregs and subsets cell percentages of all three donors exhibited stable profiles with less than $20 \%$ change from baseline, thus, indicating that stability could be extended to at least 11 days (Figure $3 \mathrm{E}-\mathrm{H}$ ). Together, these results suggest that frozen storage of lysed whole blood is contributing to improved stability as compared with storing whole blood samples at RT.

The impact of different sample storage methods on the expression of cellular markers was evaluated by analysis of MFI of several critical markers in the assay. These include surface marker CD25, intracellular markers FOXP3 and Helios. In contrast to stable FOXP3 ${ }^{+}$Tregs\%, a steady decline in FOXP3 MFI of both Tregs and FOXP3 ${ }^{+}$Tregs was observed in whole blood stored at RT. And by day 3, the majority of the donors displayed greater than $20 \%$ change from baseline (Figure 4A-B). Interestingly, Helios MFI of $\mathrm{Helios}^{+} \mathrm{FOXP}^{+}$Tregs exhibited marked stability from day 0 to 3 (Figure 4C), whereas CD25 MFI of Tregs dropped around 20\% initially in all 3 donors and then remained stable for the rest of testing period. On the other hand, when whole blood samples were lysed and stored frozen, remarkable stability was observed for MFI of FOXP3, Helios and CD25 in all three donors and beyond 3 days. As shown in Figure 4E-H, all the MFI changes from baseline values were consistently less than $15 \%$ for 11 days in all three donors. These results indicate that freezing lysed whole blood is superior in stabilizing cellular marker expression.

The stability of the absolute count of cells per $\mu$ l of Tregs and subsets in lysed frozen whole blood was evaluated and shown in Figure 5A-D. Similar to cell percentages and MFI values, the absolute counts of Tregs, FOXP3 ${ }^{+}$ Tregs, Helios ${ }^{+} \mathrm{FOXP}_{3}{ }^{+}$Tregs and $\mathrm{CD}_{4} 5 \mathrm{RO}^{+} \mathrm{FOXP}^{+}$Tregs were stable for at least 11 days. These results further exemplify that frozen storage of lysed whole blood is associated with prolonged stability of Treg populations, thus, valuable for clinical scenarios. 


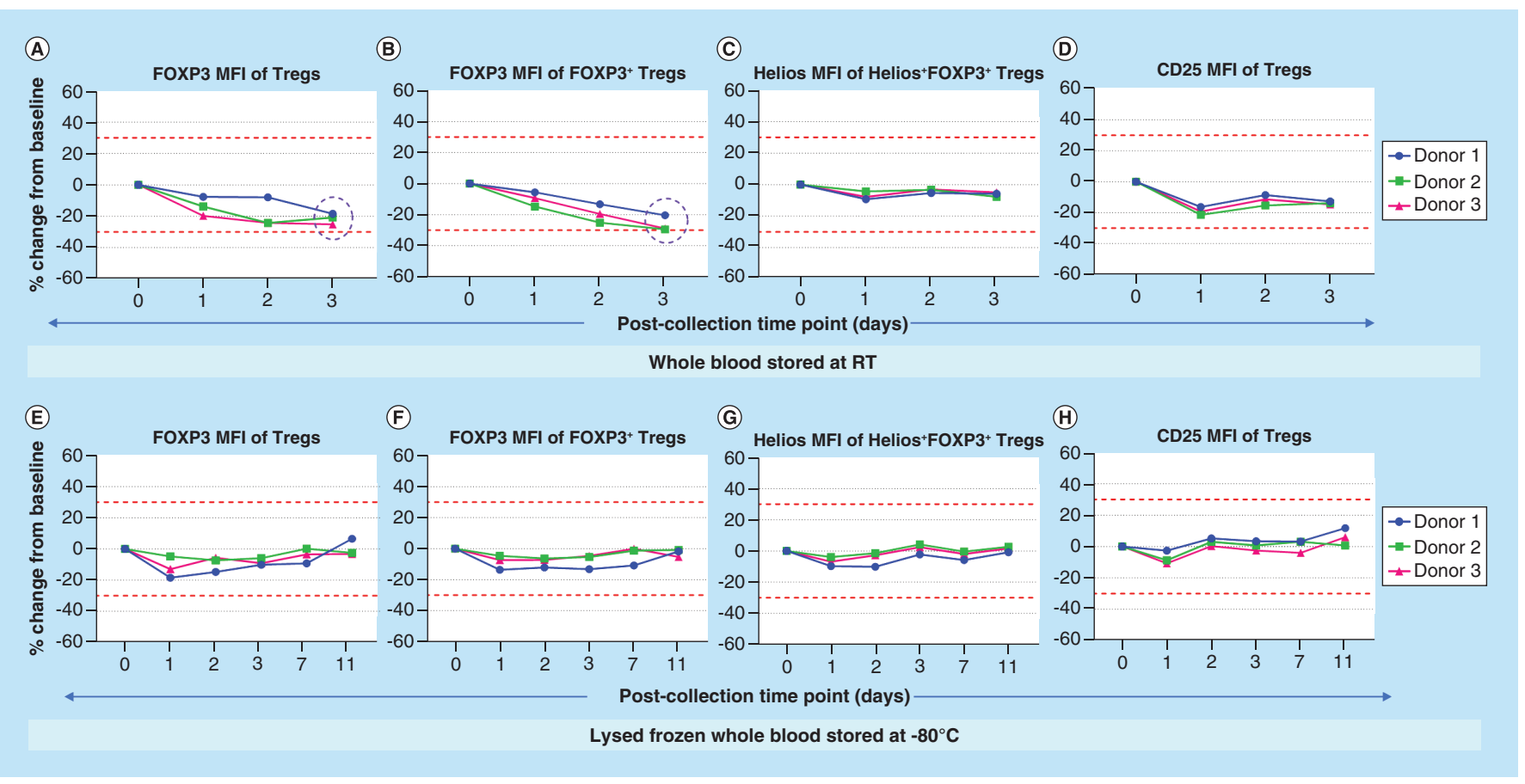

Figure 4. Comparison of stability profiles of median fluorescent intensity values. Stability was evaluated for whole blood samples at RT and lysed frozen whole blood at $-80^{\circ} \mathrm{C}, \mathrm{n}=3$. Graphs show the percent change from baseline for individual MFIs at RT (A-D) and at $-80^{\circ} \mathrm{C}$ (E-H). The X-axis represents postcollection time points. Top and bottom red lines on each graph indicate $+30 \%$ and $-30 \%$ change from baseline, respectively. Purple circles indicate change from baseline $\geq 20 \%$.

MFI: Median fluorescent intensity; RT: Room temperature.

\section{Conclusion}

Clinical flow cytometry end points are one of the key parameters that measures the outcome of a clinical trial. The typical method of sample transport is shipment of the whole blood sample after draw from the clinic to the bioanalytical laboratory at RT, a process that could take more than $24 \mathrm{~h}$ depending on the distance between the clinical sites and the testing laboratory. Since Treg-associated markers are critical to clinical research, it is apparent that the continual decline in the stability poses one of the biggest challenges. Such scenarios not only complicate sample logistics but may also pose a risk to data quality. While RT stored samples are associated with the ease of sample shipment but limited stability, it may not be adequate for clinical studies concerning Tregs and related functional markers as shown in this study and certain leukocyte subsets as observed by others [11]. To mitigate these issues, whole blood was lysed and stored frozen, and the results indicated substantially extended stability as compared with RT storage. Furthermore, this lyse freeze method could present multiple advantages such as sample centralization, batch processing, cost-effectiveness and reduced scheduling conflicts. Thus, making it desirable for clinical trials. A comprehensive validation of this method ( $\mathrm{n}=3$ additional donors) has shown that lysed frozen sample stability can be extended up to 12 days (assessment time points $0,1,2,5,7$ and 12 days; data not shown). In-study validation for longer frozen sample stability is currently ongoing. Additionally in our lab, a similar approach had been successfully applied to support a clinical trial in monitoring Tregs and associated markers such as Ki67, ICOS, Helios and CTLA-4.

\section{Future perspective}

Clinical therapies pertaining to Tregs are on the rise due to involvement of these populations in multiple pathologies. We anticipate that with the discovery of novel disease-associated biomarkers, clinical research will be heavily dependent on flow cytometry. The current study highlights one of the approaches to mitigate limited stability issues often noticed with flow assays supporting clinical trials. This approach is not just limited to the clinical studies involving Tregs but can be implemented in clinical trials that monitor other challenging cellular markers, the feasibility of which can be evaluated during method development. Furthermore, based on the clinical study requirements, samples may be lysed and frozen at the clinical sites, provided appropriate training is received by 

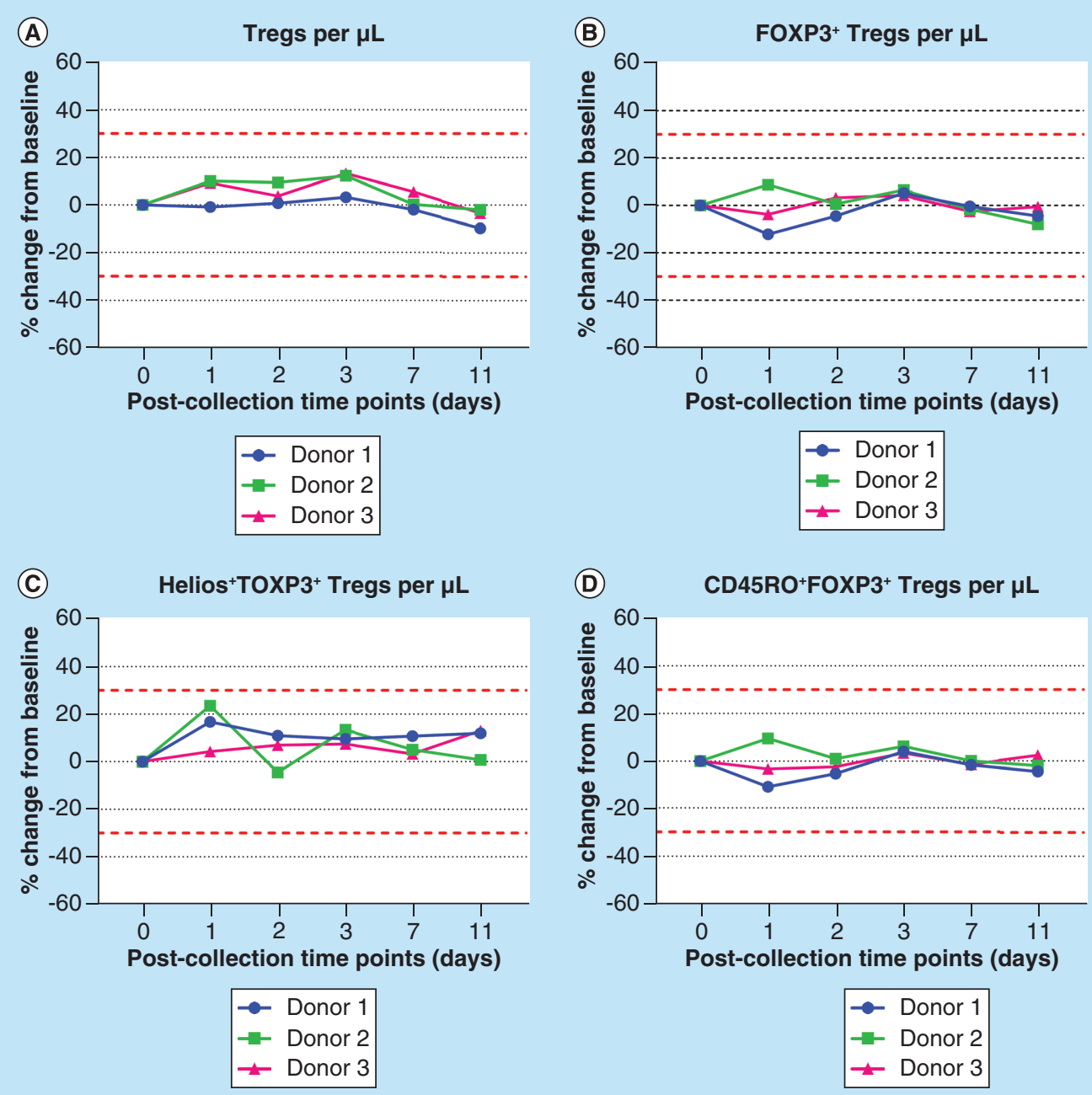

Figure 5. Stability profiles of absolute counts per $\mu \mathrm{l}$ for Tregs and subsets. Absolute counts per $\mu$ l were evaluated from lysed frozen whole blood samples, $n=3$. Each graph shows absolute counts per $\mu l$ for individual populations (A-D). The $Y$ - and $X$-axis show percent change from baseline and postcollection time points, respectively. Top and bottom red lines on each graph indicate $+30 \%$ and $-30 \%$ change from baseline, respectively.

responsible personnel. Cryopreservation of peripheral blood mononuclear cells may appear to be a comparable alternative. However, comparing to whole blood lysis and flash freezing, peripheral blood mononuclear cells isolation and cryopreservation are more tedious and technically challenging, therefore may not be feasible at every clinical site. With more clinical trials utilizing flow cytometry, innovative methods that can alleviate sample stability challenges will play a pivotal role down the road. All together, these factors will be given a lot of importance by the clinical research community as these may ultimately prove to be critical for the success of the entire clinical program.

\section{Acknowledgments}

The authors would like to acknowledge $\mathrm{H}$ Tadema for reviewing the manuscript and providing valuable comments and suggestions.

Financial \& competing interests disclosure

All authors are employees of PRA Health Sciences and have benefits of employment from the company, including stock ownership. The authors have no other relevant affiliations or financial involvement with any organization or entity with a financial interest in or financial conflict with the subject matter or materials discussed in the manuscript apart from those disclosed.

No writing assistance was utilized in the production of this manuscript. 


\section{Ethical conduct of research}

The authors state that they have obtained appropriate institutional review board approval or have followed the principles outlined in the Declaration of Helsinki for all human or animal experimental investigations. In addition, for investigations involving human subjects, informed consent has been obtained from the participants involved.

\section{Executive summary}

- Tregs are a distinct and rare subset of $\mathrm{CD} 4^{+} \mathrm{T}$ cells that are able to modulate immune responses.

- Flow cytometry assays that can effectively enumerate Treg populations play an important role toward the success of Treg-based clinical studies.

- A major challenge that encompasses the development of clinical flow cytometry assays is sample stability, especially the assays that involve rare populations such as Tregs.

- Sodium heparin proved to be the candidate sample collection matrix that can support both optimal assay performance and sample stability.

- Whole blood samples showed continuous loss of Tregs stability over time when stored at room temperature. On the contrary, our approach to lyse freeze whole blood maintained Treg stability for extended periods of time.

- If the whole blood lysis and flash freeze are to be performed at clinical sites, responsible personnel should be fully trained and should demonstrate competency through an established qualification program.

- Lyse freeze method is associated with logistical advantages along with being cost-effective to both sponsors and clinical research organizations.

\section{Open access}

This work is licensed under the Attribution-NonCommercial-NoDerivatives 4.0 Unported License. To view a copy of this license, visit http://creativecommons.org/licenses/by-nc-nd/4.0/

\section{References}

1. Zhao H, Liao X, Kang Y. Tregs: where we are and what comes next? Front. Immunol. 8, 1578 (2017).

2. Romano M, Fanelli G, Albany CJ, Giganti G, Lombardi G. Past, present, and future of regulatory T cell therapy in transplantation and autoimmunity. Front. Immunol. 10, 43 (2019).

3. Dominguez-Villar M, Hafler DA. Regulatory T cells in autoimmune disease. Nat. Immunol. 19(7), 665-673 (2018).

4. Singer BD, King LS, D’Alessio FR. Regulatory T cells as immunotherapy. Front. Immunol. 5, 46 (2014).

5. Duggleby R, Danby RD, Madrigal JA, Saudemont A. Clinical grade regulatory CD $4\left(^{+}\right) \mathrm{T}$ cells (Tregs): moving toward cellular-based immunomodulatory therapies. Front. Immunol. 9, 252 (2018).

6. Ye C, Brand D, Zheng SG. Targeting IL-2: an unexpected effect in treating immunological diseases. Signal Transduct. Target. Ther. 3, 2 (2018).

7. Fanton C, Dixit N, Siddhanti S et al. Selective induction of functional regulatory T-cells in healthy volunteers by NKTR-358, a novel IL-2 conjugate Treg stimulator, in development for the treatment of autoimmune diseases. Presented at: ACR/ARP Annual Meeting. Atlanta, GA, USA, 8-13 November 2019.

8. Strate BVD, Longdin R, Geerlings $\mathrm{M}$ et al. Best practices in performing flow cytometry in a regulated environment: feedback from experience within the European Bioanalysis Forum. Bioanalysis 9(16), 1253-1264 (2017).

9. Brown L, Green CL, Jones $\mathrm{N}$ et al. Recommendations for the evaluation of specimen stability for flow cytometric testing during drug development. J. Immunol. Methods 418, 1-8 (2015).

10. Plate MM, Louzao R, Steele PM et al. Evaluation of the blood stabilizers TransFix and Cyto-Chex BCT for low-cost CD4 T-cell methodologies. Viral Immunol. 22(5), 329-332 (2009).

11. Diks AM, Bonroy C, Teodosio C et al. Impact of blood storage and sample handling on quality of high dimensional flow cytometric data in multicenter clinical research. J. Immunol. Methods 475, 112616 (2019).

12. Nettenstrom L, Alderson K, Raschke EE et al. An optimized multi-parameter flow cytometry protocol for human T regulatory cell analysis on fresh and viably frozen cells, correlation with epigenetic analysis, and comparison of cord and adult blood. J. Immunol. Methods 387, 81-88 (2013)

13. Santegoets SJ, Dijkgraaf EM, Battaglia A et al. Monitoring regulatory T cells in clinical samples: consensus on an essential marker set and gating strategy for regulatory T cell analysis by flow cytometry. Cancer Immunol. Immunother. 64(10), 1271-1286 (2015).

14. Pitoiset F, Barbié M, Monneret G et al. Standardized flow cytometry procedure for the monitoring of regulatory $\mathrm{T}$ cells in clinical trials. Cytometry B Clin. Cytom. 94(5), 777-782 (2018).

15. Chaudhary B, Elkord E. Regulatory T cells in the tumor microenvironment and cancer progression: role and therapeutic targeting. Vaccines (Basel) 4(3), 28 (2016). 
16. Rosenblum MD, Way SS, Abbas AK. Regulatory T cell memory. Nat. Rev. Immunol. 16(2), 90-101 (2016).

17. Mathew JM, H-Voss J, LeFever A et al. A Phase I clinical trial with ex vivo expanded recipient regulatory $\mathrm{T}$ cells in living donor kidney transplants. Sci. Rep. 8, 7428 (2018).

18. Getnet D, Grosso JF, Goldberg MV et al. A role for the transcription factor Helios in human $\mathrm{CD} 4\left({ }^{+}\right) \mathrm{CD} 25\left(^{+}\right)$regulatory T cells. Mol. Immunol. 47(7-8), 1595-1600 (2010).

19. Warrino DE, DeGennaro LJ, Hanson M, Swindells S, Pirruccello SJ, Ryan WL. Stabilization of white blood cells and immunologic markers for extended analysis using flow cytometry. J. Immunol. Methods 305(2), 107-119 (2005). 
